\title{
Case Scenario about General Anesthesia Management of Patient with Pulmonary Vein Stenosis underwent Balloon Dilatation and Stent Implantation
}

\author{
Fangzhou Li, M.D., Guoyan Qiao, M.D., Peng Liang, M.D. , Jin Liu, M.D., Ph.D. \\ From Department of Anesthesiology, West China Hospital, Sichuan University, Chengdu, P. R. China
}

Background : Pulmonary vein stenosis (PVS) is a rare Discussion: Acute pulmonary arteriovenous rupture hemorrhage, cardiovascular deformity that can lead to high mortality. Patients acute increase in right heart after-load, acute right heart failure, frequently experience multiple complications such as hemoptysis, transient ST segment elevation, blood clot detachment, pulmonary vein pulmonary hypertension, bronchial venous rupture and cardiac laceration-induced hemothorax, left atrial opening rupture of pulmonary insufficiency. Currently, pulmonary vein stenosis balloon dilatation vein-induced acute cardiac tamponade and left heart failure may occur (stent implantation) is the only treatment, and this can be performed during and after balloon dilatation. Simultaneously, airway pressure under local or general anesthesia. However, a case report on the should be monitored closely and prompt suction is necessary to remove general anesthesia management of PVS has not been previously the airway secretion, sustain the respiratory tract unobstructed and reported. In this case report, we discuss anesthetic considerations maintain the normal pCO2 level. In the meanwhile, the ECMO should in patients with PVS, focusing specifically on perioperative airway be immediately performed once the occurrence of any of these and circulatory management as well as the risk evaluation, and the complications, such as pulmonary vein rupture, pericardial tamponade. appropriate effective management of all potential complications And the patient should be transferred to the thoracotomy immediately. intraoperatively.

Case Report : A 58-year-old male patient was admitted due to coughing rusty sputum for over two years as well as experiencing dyspnea upon exertion and chest distress after exercise for one year. The patient received circumferential pulmonary vein isolation two times due to atrial fibrillation two years ago and one year ago in a different hospital. He gradually developed coughing rusty sputum and exertional dyspnea after surgery and proposed to undergo pulmonary vein stenosis stent implantation under general anesthesia. No abnormality was seen in the lung, heart and abdomen physical examination. TTE revealed slightly enlarged RV, tricuspid regurgitation (mild), pulmonary hypertension (moderatesevere), $P G=71 \mathrm{mmHg}, P A P=74 \mathrm{mmHg}$. CT revealed scattered ground glass degeneration and interstitial edema in both lungs. Stenosis or occlusion in the initial segments of LSPV,LIPV and RIPV. The ECG displayed the suspicious pulmonary embolism ECG $\left(S_{1} Q_{\| I} T_{\|||}\right.$signs). The diagnosis included pulmonary vein stenosis, pulmonary hypertension (secondary) and underwent balloon dilatation and stent implantation.

\section{Surgical experience and anesthesia management}

The vital signs in OR:HR $96 \mathrm{bpm}$, NBP $91 / 56 \mathrm{mmHg}$, RR 20 tpm, $\mathrm{S}_{\mathrm{p}} \mathrm{O}_{2} 92 \%$ (99\% after $80 \% \mathrm{O}_{2}$ inhalation). ABP, CVC,surface defibrillation electrodes were prepared, ECMO was standby. Noradrenaline was injected at an initial infusion of $0.03 \mu \mathrm{g} / \mathrm{kg} \mathrm{min}^{-1}$ during induction to inhibit the peripheral vascular dilation induced by general anesthetics. Propofol and remifentanil were for intraoperative maintenance. We monitored the pressure gradient at different sites(Table 1).Before balloon dilatation, epinephrine $(0.05$ $\mu \mathrm{g} / \mathrm{kg} \mathrm{min}^{-1}$ ) was added and noradrenaline was reduced. The LSPV was selected as the first site of intervention, which was the most stenosis and with almost complete occlusion as well as mild interference to the blood circulation. The furosemide $(5 \mathrm{mg})$ was twice administrated intravenously before the procedure of LSPV and LIPV balloon dilatation was performed in order to prevent the preload of left heart increasing dramatically after pulmonary vein blood flow recovery. Three metal stents (I.D. $=18 \mathrm{~mm}, 10 \mathrm{~mm}$, $10 \mathrm{~mm}$ ) were implanted respectively after each balloon dilatation. Vital signs were maintained stable intraoperatively, and the surgery had lasted for 4 hours. During the surgery, the fluid supplement was $900 \mathrm{ml}$, and the urine output was $1000 \mathrm{ml}$. Postoperative TTE revealed no pericardial effusion, no accelerated pulmonary vein stent blood flow, mild tricuspid regurgitation and normal left ventricular systolic function. The vasoactive agents were gradually discontinued, and the tracheal tube was extubated after the patient awake. The patient was transferred to cardiac care unit (CCU) and discharged seven days later after rehabilitation. Furthermore, long-term pulmonary vein stenosis will induce to the reduced left heart preload, which may be further reduced during the balloon dilatation, resulting in decreased cardiac output and hypotension. Therefore, intraoperative hypotension should be managed temporarily using vasoactive prescriptions. Pulmonary ventilation resistance should be closely monitored during and after the stent implantation, so as to prevent the sharp decreasing of the right heart after-load, which may result in the excessive pulmonary perfusion, leading to acute and severe pulmonary effusion. Meanwhile, the respiratory parameters should be well set, and the positive endexpiratory pressure should be properly added to reduce the possibilities of pulmonary effusion. The left heart function should be carefully monitored by using cardiogram ultrasonic to avoid significant increasing of the left heart preload after dilatation, which may induce acute left heart failure and malignant arrhythmia. Specifically, small dose applications of epinephrine and furosemide have the ability to enhance myocardial contractility, reduce the blood volume and prevent the occurrence of acute heart failure in this period. In addition, ECMO should be placed timely when respiratory circulation cannot be maintained intraoperatively and the regular medications failed to ameliorate the symptoms.

Table 1

The video of LSPV

\begin{tabular}{|l|l|l|}
\hline & $\begin{array}{l}\text { Pressure (mmHg) } \\
\text { before balloon } \\
\text { dilatation }\end{array}$ & $\begin{array}{l}\text { Pressure (mmHg) } \\
\text { after balloon } \\
\text { dilatation }\end{array}$ \\
\hline Site & $\begin{array}{l}\text { Systolic/Mean/Diast } \\
\text { olic }\end{array}$ & $\begin{array}{l}\text { Systolic/Mean/Dia } \\
\text { stolic }\end{array}$ \\
\hline PA & $41 / 24 / 16$ & $45 / 24 / 13$ \\
\hline LA & $12 / 6 / 3$ & $17 / 10 / 6$ \\
\hline RV & $45 / 15 / 2$ & $44 / 15 / 1$ \\
\hline SVC & $10 / 7 / 5$ & $9 / 6 / 4$ \\
\hline LSPV & $22 / 17 / 14$ & $19 / 13 / 10$ \\
\hline LSPA & $37 / 19 / 10$ & $35 / 19 / 12$ \\
\hline
\end{tabular}

$\delta P=P V-L A$

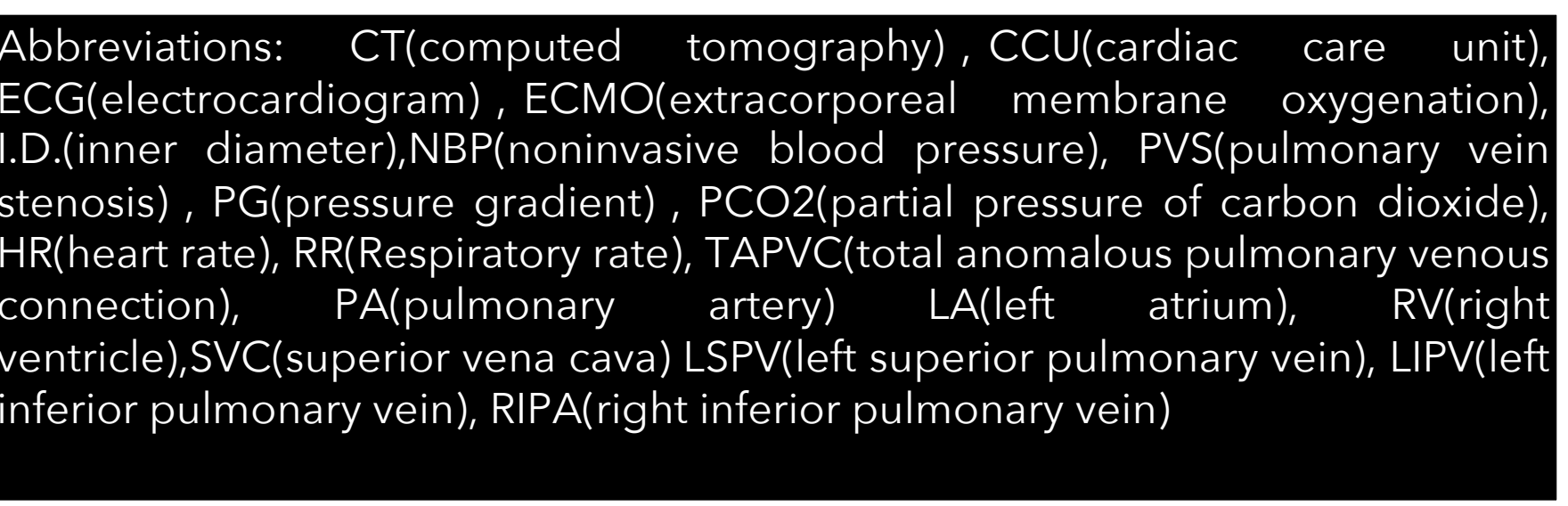

Learning points:

1.Additional cautions and preparations including vasoactive drugs, defibrillation electrode in vitro and the ECMO.

2. Furosemide used before balloon dilatation to prevent the preload of left heart increasing dramatically after pulmonary vein blood flow recovery. 